FORMATLON Formation emploi

Revue française de sciences sociales

134 | Avril-Juin

Pêle-mêle

\title{
Le rôle des caractéristiques des établissements dans le décrochage scolaire
}

L'exemple de l'académie d'Aix-Marseille

The role of school characteristics on the school dropout. The example of the

Academy of Aix-Marseille

Die Rolle der besonderen Merkmale einer Schuleinrichtung beim Schulabbruch

am Beispiel der Académie (Schulbezirk) Aix-Marseille

El papel de las características de los establecimientos en la deserción escolar. El

ejemplo de la academia de Aix-Marseille

\section{EL-Mahdi Khouaja et Stéphanie Moullet}

\section{OpenEdition}

Journals

Édition électronique

URL : http://journals.openedition.org/formationemploi/4713

DOI : 10.4000/formationemploi.4713

ISSN : 2107-0946

Éditeur

La Documentation française

Édition imprimée

Date de publication : 21 juin 2016

Pagination : 7-26

ISSN : 0759-6340

Référence électronique

EL-Mahdi Khouaja et Stéphanie Moullet, «Le rôle des caractéristiques des établissements dans le décrochage scolaire », Formation emploi [En ligne], 134 | Avril-Juin, mis en ligne le 22 juin 2016, consulté le 30 octobre 2020. URL : http://journals.openedition.org/formationemploi/4713 ; DOI : https://doi.org/10.4000/formationemploi.4713 


\title{
Le rôle des caractéristiques des établissements dans le décrochage scolaire
}

\section{L'exemple de l'académie d'Aix-Marseille}

\begin{abstract}
EL-MaHDI KHOUAJA
Economiste, doctorant à Aix-Marseille université, CNRS, Laboratoire d'économie et de sociologie du travail (LEST- UMR 7317). Il occupe la fonction de coordonnateur-bassins de

la Mission de Lutte contre le Décrochage Scolaire (MLDS) de l'académie d'Aix-Marseille
\end{abstract}

StÉPHANIE Moullet Economiste, maîtresse de conférences à Aix-Marseille université, CNRS, Laboratoire d'économie et de sociologie du travail (LEST-UMR 7317), Institut régional du travail

d'Aix-en-Provence

Résumé

Le rôle des caractéristiques des établissements dans le décrochage scolaire. L'exemple de l'académie d'Aix-Marseille

Cette contribution propose des portraits type des décrocheurs à partir de données sur l'académie d'Aix-Marseille. L'investigation prend également en compte le rôle des caractéristiques des établissements scolaires. L'effet "d'établissement " apparaît plus important pour les lycées généraux et technologiques que pour les lycées professionnels ; alors même que le décrochage scolaire est sensiblement plus faible dans les premiers que dans les seconds.

Mots clés : établissement d'enseignement, abandon des études, origine sociale, famille, cheminement scolaire, économétrie

Abstract

The role of school characteristics on the school dropout. The example of the Academy of Aix-Marseille

This article aims to establish the profile of dropouts using data on the Academy (Education Authority) of Aix-Marseille. We also examine the characteristics of schools to explain their role in the dropout. The school effect appears more important for 
general and technical secondary education institutions for vocational schools; even as school dropout is significantly lower in the former than in the latter.

Keywords: educational institution, drop out, social origin, family, school paths, econometrics

Journal of Economic Literature: C 21, I 21

Traduction : Auteur.e.s

La lutte contre le décrochage scolaire est l'une des priorités de la loi d'orientation et de programmation pour la refondation de l'école de la République de juillet $2013^{1}$. L’objectif est de diminuer de moitié, d'ici 2017, le nombre de jeunes sortant du système éducatif sans diplôme, qui s'élève aujourd'hui à 140000 jeunes (Weixler, 2014). Cet objectif correspond également à l'une des priorités de la stratégie "Europe 2020 » : réduire à moins de $10 \%$ le taux d'abandon scolaire (Conseil de l'UE, 2009).

Le décrochage scolaire reflète des enjeux importants en matière d'employabilité des jeunes. En France et en 2013, le taux de chômage des sortants sans diplôme atteint 50 \%, contre $23 \%$ pour l'ensemble des jeunes, selon l'enquête Génération 2010 du Céreq. Les décrocheurs occupent aussi des emplois dont la qualité est dégradée relativement à celle de l'emploi des diplômés (Barret et al., 2014).

Par ailleurs, des travaux ont montré que les caractéristiques sociodémographiques des jeunes décrocheurs qui influent sur leur décrochage peuvent également être corrélées avec des comportements à risque en matière de délinquance (Sweeten et al., 2009); pour autant, une sortie précoce du système scolaire ne se traduit pas systématiquement par une entrée dans la délinquance (Esterle-Hedibel, 2003a). Reste que la maîtrise de l'ensemble de ces enjeux passe par la connaissance des facteurs de décrochage, individuels mais aussi contextuels. Il s'agit en effet de prévenir les risques d'abandon scolaire et les conséquences qui en découlent.

En mars 2013, l'académie d'Aix-Marseille présente un taux de décrochage de $9.3 \%$, proche de la moyenne nationale. Cette académie scolarise $4.4 \%$ des élèves français et $4.1 \%$ des décrocheurs sont issus de son territoire. L'hétérogénéité sociale à l'intérieur et entre les établissements scolaires d'une académie est forte (MENESR, 2014) et des problématiques spécifiques à chaque territoire existent (Boudesseul et al., 2014). Il y a donc un intérêt à conduire des analyses sur un territoire donné. La pertinence de l'analyse pour l'académie d'Aix-Marseille tient non seulement à sa relative représentativité de la situation nationale (même ampleur du phénomène, même caractéristiques socioéconomiques du public sco-

1. Notre recherche s'inscrit dans le cadre d'un programme financé par l'Agence nationale de la recherche : Territoires et décrochages scolaires (TEDS) ANR-14-CE30-0009-01. 
larisé dans l'enseignement secondaire et une dotation horaire par élève équivalente à la moyenne nationale) mais aussi à la qualité des données disponibles. Ces dernières ont en effet été construites et rendues "fiables ", c'est-à-dire apurées des "faux » décrocheurs par des contrôles successifs ; cela a été rendu possible grâce à une collaboration avec la direction de l'analyse, des études et de la communication (DAEC) du rectorat.

Cet article s'attache, dans un premier temps, à dresser le portrait type des décrocheurs de l'académie d'Aix-Marseille, en 2012-2013. Dans un second temps, à partir d'une approche multiniveaux, l'analyse modélise les effets de l'environnement scolaire sur les élèves. L'investigation porte sur les caractéristiques des établissements scolaires fréquentés pour expliquer, au-delà des déterminants individuels aujourd'hui bien documentés, leur rôle sur le risque de décrochage. La taille de l'établissement par exemple, sa composition sociale ou encore le niveau des élèves qu'il scolarise ont-ils un impact propre sur le risque de décrocher?

Après l'exposé des facteurs de décrochage largement identifiés dans la littérature, notamment au niveau individuel, nous présentons les données utilisées et leur originalité. Les profils-types de décrocheurs de l'académie d'Aix-Marseille sont alors ensuite établis. Enfin, le rôle des caractéristiques du dernier établissement scolaire fréquenté sur le risque de décrochage est précisément étudié. Nous concluons par les implications et limites des résultats.

\section{Pour la littérature, le décrochage est un processus complexe et multifactoriel}

Depuis plus de quarante ans, un consensus se dégage de la littérature sur le caractère processuel et multifactoriel du décrochage scolaire ${ }^{2}$. Il s'agit d'un processus complexe qui combine des facteurs individuels, sociaux et contextuels, et qui ne se résume pas au simple constat objectif d'une situation de décrochage (Bernard, 2011). Cette situation met un terme à un long malentendu sociocognitif entre les attentes de l'institution scolaire et celles de l'élève (Bonnéry, 2007), au décalage entre des logiques sociales et des logiques scolaires qui s'opèrent à l'intérieur de l'école (Millet et Thin, 2005), faisant du décrochage scolaire l'expression concomitante des problématiques scolaires et sociales (Glasmann et Oeuvrard, 2004).

Les nombreuses recherches portant sur les facteurs de décrochage scolaire séparent les facteurs individuels liés à l'élève, des facteurs qui caractérisent l'environnement scolaire et des facteurs sociaux qui relèvent du milieu familial (Rumberger, 2004). Le sexe de l'élève, la faible performance scolaire aux évaluations disciplinaires (Dardier et al., 2013 ; Afsa,

2. Des travaux ont identifié au moins 228 facteurs d'influence sur le processus d'apprentissage et la scolarité des élèves (voir Wang et al., 1990). 
2013), le redoublement au primaire ou au collège (Broccolichi et Larguèze, 1996) sont des facteurs "annonciateurs" de décrochage scolaire. L'élève "à risque " se caractérise aussi par un faible degré d'estime de soi (Parker et Asher, 1987) ; il vit dans un environnement dominé par de faibles aspirations scolaires et professionnelles (Vitaro et al., 2001 ; Archambault et Janosz, 2009 ; Dardier et al., 2013).

\section{Encadré 1 : Définir et mesurer le décrochage scolaire}

Selon l'article 313-7 du code de l'éducation créé par la loi n²009-1437 du 24 novembre 2009 et le décret $n^{\circ} 2010-1781$ du 31 décembre 2010, un décrocheur est un jeune de plus de 16 ans, sorti du système scolaire et non inscrit en apprentissage, n'ayant pas obtenu un baccalauréat général ou un diplôme à finalité professionnelle enregistré au Répertoire national des certifications professionnelles et classé au niveau V ou IV (c'est à dire aux CAP-BEP respectivement Certificat d'aptitude professionnelle et Brevet d'études professionnelles - ou Baccalauréat) de la nomenclature interministérielle des niveaux de formation. Sont aussi considérés comme décrocheurs, les jeunes qui ont obtenu un diplôme de niveau V (CAP-BEP) et qui ont échoué dans I'obtention d'un diplôme de niveau IV. Cette définition " officielle » fournit une première estimation du nombre de jeunes décrocheurs grâce au système interministériel d'échange d'information (SIEI) ; ce dernier permet la constitution d'une base de données de décrocheurs suite à un croisement de bases issues du ministère de l'Education nationale, celui de l'Agriculture pour l'enseignement agricole et le ministère du Travail pour les données des Missions Locales. En mars 2012, le SIEl a repéré 229877 décrocheurs en France (Marc, 2013).

La conception du décrochage scolaire admise ici est celle d'un acte constaté à un instant donné (tout en étant l'aboutissement d'un processus conjuguant l'interaction de nombreux facteurs) et par conséquent mesurable selon des critères objectifs. Si l'Education nationale a son propre outil de repérage des décrocheurs, d'autres indicateurs qui se rapprochent de cette conception du décrochage sont également retenus selon le champ de compétences des institutions qui les produisent.

Le taux des sortants sans diplôme mesure, quant à lui, la proportion des jeunes sortant de la formation initiale sans CAP, BEP ou niveau supérieur ou uniquement avec le brevet des collèges, quel que soit leur âge. Évalué à partir des enquêtes Emploi de l'Insee, ce taux est une estimation basée sur les flux des sortants de la formation initiale durant trois années. On compte 122000 sortants sans diplôme, chiffre estimé sur une population moyenne de 713000 jeunes sortants de la formation initiale en 2008, 2009 et 2010. Les enquêtes d'insertion (Enquêtes Génération) du Céreq permettent aussi d'identifier le nombre de jeunes sortants sans diplôme. L'enquête la plus récente rapporte que 112000 jeunes sont dans cette situation et ils représentent $16 \%$ de l'ensemble des sortants du système éducatif en 2010.

Un autre indicateur, au niveau européen, est le taux des sortants précoces qui mesure la part des 18-24 ans sans diplôme ou avec uniquement un brevet du collège et qui ne sont pas en formation au moment de l'enquête. En France et en 2014, ce taux s'élève à $8.5 \%$, en-dessous de l'objectif fixé par l'UE (9.5\%). A la différence du taux des sortants sans diplôme, ce taux prend en compte les jeunes ayant bénéficié d'une formation en dehors du cadre de la formation initiale (reprise d'étude, contrat de professionnalisation, formation prescrite par la Mission Locale,...). 
Quant au contexte scolaire, certains facteurs pourraient contribuer à l'augmentation du décrochage. La taille de l'établissement scolaire, le leadership pédagogique du chef d'établissement et la coopération entre les enseignants (Dumay et Depriez, 2004) ont des effets sur la performance scolaire des élèves, notamment pour ceux qui sont issus d'un milieu modeste (Afsa, 2013), effets qui pourraient, " par ricochet ", agir sur de décrochage scolaire. Des recherches ont souligné l'importance des caractéristiques de l'établissement, comme la composition sociale du public accueilli, le taux d'encadrement, les caractéristiques des enseignants, la filière proposée ou le secteur de l'établissement (public ou privé) ainsi que ce qui relève des pratiques éducatives, sur le risque de décrochage (Bryk et Thum, 1989 ; McNeal, 1997 ; Millet et Thin, 2005).

Enfin, l'ensemble des travaux s'accordent sur la force prédictive de certaines caractéristiques du milieu familial. Une situation économique fragile (Bénabou et Tirole, 2004), un faible niveau d'études des parents, notamment celui de la mère (Caille, 2000), et la monoparentalité de la famille (Dardier et al., 2013) influent sur le décrochage. Le rôle important des pratiques éducatives dans le milieu familial (Fortin et $a l ., 2004$ ), la langue parlée au domicile (autre que la langue d'enseignement scolaire) et le faible niveau d'équipement du logement en matière de matériels aidant à l'apprentissage, font également partie des facteurs familiaux du décrochage scolaire (Rumberger, 1987).

De cette revue de la littérature, nous retenons que la performance scolaire, l'environnement familial dans sa dimension socio-économique et le contexte scolaire correspondent à des situations associées exposant davantage au décrochage scolaire. Nous cherchons à saisir ces différentes dimensions au travers des variables disponibles dans les données utilisées ici.

\section{Une base de données exhaustive}

Les données du système interministériel d'échange d'information (SIEI) ne recensent que les jeunes décrocheurs et offrent peu de variables ${ }^{3}$. Une convention d'échange d'information avec l'académie d'Aix-Marseille a permis la construction d'une base de données pour répondre aux besoins de cette recherche.

Ces données regroupent des variables caractérisant l'élève, la famille et l'école à travers un ensemble d'indicateurs. A partir des fichiers de la base élèves académique (BEA) des deux années scolaires 2011-2012 et 2012-2013, on identifie les élèves ayant décroché de la formation initiale dans l'académie d'Aix-Marseille.

3. Les fichiers du SIEI sont régis par les articles 8 et 9 de la loi informatique et libertés, ils ne peuvent contenir aucune donnée relative à des infractions, à la santé, à la nationalité, ou à la situation sociale ou familiale de l'élève. 
Ces décrocheurs sont donc les élèves, âgés de 16 ans ou plus en mars 2013, scolarisés en octobre 2011 dans l'enseignement public et privé sous contrat et qui, en mars 2013, n'étaient plus scolarisés, ni n'avaient intégré un CFA (centre de formation d'apprentis) et qui, à la session 2012, n'avaient pas obtenu de diplôme sanctionnant leur cursus (Bac, BEP, CAP).

Au final, les données constituées comptent 108203 élèves âgés de 16 ans et plus en mars 2013, dont 10045 décocheurs, n'ayant pas intégré un CFA et n'ayant pas obtenu un diplôme (Bac, BEP, CAP), soit un taux de décrochage de $9.3 \% .^{4}$.

Nos données couvrent alors les principales dimensions des trois catégories de facteurs retenues. Elles permettent de caractériser : la performance scolaire (résultat et note au DNB, retard scolaire), le parcours scolaire (classe et établissement scolaire d'origines), l'environnement socio-économique familial (sexe de l'élève, CSP - catégorie sociale et professionnelle des deux responsables légaux - monoparentalité et bourses attribuées sur critères sociaux) et des variables concernant les établissements scolaires (taille, secteur d'enseignement, moyenne au DNB des élèves scolarisés, caractéristiques du public accueilli, encadrement, ...). Ces données concernent à la fois les décrocheurs et les non-décrocheurs.

\section{Portraits-types des décrocheurs 3 de l'académie d'Aix-Marseille}

En mars 2013, le taux de décrochage pour l'académie d'Aix-Marseille est relativement comparable à celui observé dans les quatre départements qui la composent : $9.1 \%$ pour le département des Alpes-de-Haute-Provence, $9.4 \%$ pour celui des Bouches-duRhône, 8.2 \% pour les Hautes-Alpes et $9.1 \%$ pour le Vaucluse.

L'enseignement public affiche un taux de décrochage supérieur au secteur d'enseignement privé $(9.6 \%$ contre $8 \%)$.

Le décrochage scolaire est sensiblement moindre pour le second cycle de l'enseignement général et technologique (G\&T-5.3\%) que pour le second cycle d'enseignement professionnel (LP-16.9\%). Quant au premier cycle de l'enseignement secondaire, le taux est de $12.1 \%$ supérieur à la moyenne académique.

4. À titre comparatif, en octobre 2013, le SIEI recensait 8076 jeunes ayant décroché de la formation initiale, pour une population de référence âgée de 16 ans et plus qui s'élève à 91255 , soit un taux $8.9 \%$. La différence peut s'expliquer par le changement de la population de référence induit par l'effet mécanique du critère «âge des élèves " entre les mois de mars et octobre 2013, par le caractère inter académiques des listes SIEI qui permet d'identifier des élèves scolarisés dans d'autres académies, mais aussi par la marge d'erreur causée par l'existence de doublons (faux décrocheurs) dans le SIEI et dans le fichier construit. 
Cette sur-représentativité des décrocheurs dans le premier cycle de l'enseignement secondaire tient au fait que la population ciblée par la mesure du décrochage intègre les élèves de 16 ans et plus, ceux qui sont scolarisés dans ce cycle, repérés comme décrocheurs, étant plus âgés que la moyenne (plus " en retard ") et donc davantage sujets au décrochage scolaire.

Comparer les élèves décrocheurs aux non-décrocheurs fournit quelques indications sur leurs profils scolaires et sociaux. Les premiers se caractérisent par une surreprésentation des retards scolaires puisque quatre décrocheurs sur cinq ont au moins un an de retard en 2011-2012, contre moins d'un élève sur deux chez les non-décrocheurs. Le passage et la réussite aux épreuves du DNB séparent nettement les deux catégories : $62 \%$ des décrocheurs ne sont pas inscrits ou ont passé sans succès les épreuves du DNB, contre $22 \%$ chez les élèves non-décrocheurs ; $50 \%$ de ces derniers ont réussi les épreuves en obtenant une moyenne générale supérieure à 10/20, contre $17 \%$ chez les élèves décrocheurs.

Les élèves boursiers sont surreprésentés chez les décrocheurs (34\% contre $26 \%$ ), de même que les élèves issus des familles monoparentales (24\% contre $15 \%$ ). La catégorie sociale et professionnelle du ménage distingue également et fortement les deux groupes d'élèves : $52 \%$ des décrocheurs sont issus d'un milieu "défavorisé ", contre $33 \%$ des non-décrocheurs.

Des profils-types des décrocheurs sont ici établis en fonction des variables scolaires (classe fréquentée, retard scolaire et réussite au DNB) et des variables socio-économiques qui médiatisent l'environnement familial de l'élève (bourse d'étude, la PCS de la famille et la monoparentalité). En termes de méthode, la typologie est obtenue à partir d'une classification ascendante hiérarchique basée sur une analyse des correspondances multiples $(\mathrm{ACM})$ des variables retenues ${ }^{6}$. Au final, on distingue quatre groupes dans la population des décrocheurs.

\subsection{Les décrocheurs « inattendus »}

Cette catégorie représente $28 \%$ des élèves décrocheurs ; le décrochage scolaire y est inattendu car les profils de ces jeunes ressemblent à ceux des élèves non décrocheurs. Filles et garçons y sont quasi également représentés. Il s'agit essentiellement d'élèves scolarisés dans le cycle d'enseignement G\&T (87\%). Au niveau de la performance

\footnotetext{
5. Sont considérés comme " défavorisés » les élèves dont la profession de la personne de référence (en général le père est : ouvrier qualifié ou non qualifié, ouvrier agricole, retraité employé ou ouvrier, chômeur n’ayant jamais travaillé, personne sans activité professionnelle ou dont l'activité est inconnue ou sans objet.

6. L'ACM permet la projection des modalités des variables retenues sur les trois premiers axes factoriels et résume $25.1 \%$ de la variance totale. La Classification Ascendante Hiérarchique des 10045 décrocheurs classe les individus dont les projections sur les deux premiers axes factoriels expliquent $18.4 \%$ de la variance totale. Cette part de la variance expliquée est relativement élevée compte tenu du nombre d'individus et de variables mobilisées (soit 7).
} 
scolaire, ce sont des élèves majoritairement sans retard scolaire (55\%) ou seulement avec un an de retard (37\%). Les élèves ayant obtenu le DNB avec ou sans la moyenne représentent $81 \%$ des élèves ( $51 \%$ avec la moyenne). On y compte des élèves non boursiers à $85 \%$, issus de familles non monoparentales à $85 \%$ et non défavorisées à $78 \%$. Le premier responsable légal de l'élève occupe un emploi dans $84 \%$ des situations, alors que le second responsable légal occupe un emploi dans $50 \%$ des cas. Enfin, les familles avec enfant unique sont majoritaires dans cette catégorie de décrocheurs.

\subsection{Les décrocheurs « désengagés»}

Il s'agit pour l'essentiel des décrocheurs qualifiés mais non diplômés. Ce groupe représente $32 \%$ des élèves décrocheurs, parmi lesquels $55 \%$ sont des garçons. Ce sont des jeunes qui vont rater leur " atterrissage " menant à la certification. Dans $80 \%$ des cas, ces jeunes étaient scolarisés dans l'enseignement professionnel (62\% pour le Bac Pro 3 ans et $17 \%$ pour le CAP en 2 ans, majoritairement en année terminale). Au niveau de la performance scolaire, $95 \%$ des élèves ont au moins un an de retard scolaire. $60 \%$ n'ont pas obtenu ou présenté le DNB, $33 \%$ l'ont obtenu avec une moyenne inférieure à 10/20. Les boursiers sont représentés à hauteur de $45 \%$. Les décrocheurs issus de familles non monoparentales représentent $72 \%$ des jeunes et $63 \%$ sont issus des familles non défavorisées. $58 \%$ des élèves décrocheurs de cette catégorie ont un responsable légal qui occupe un emploi ( $26 \%$ seulement pour le second responsable).

\subsection{Les décrocheurs « désaffectés »}

Ils représentent $25 \%$ des élèves décrocheurs. Ce sont des jeunes qui ont raté la transition vers l'enseignement professionnel. À la différence de la catégorie précédente, celle-ci rassemble des élèves scolarisés dans l'enseignement professionnel (58\%) et de la $3^{\text {ème }}$ générale du $1^{\text {er }}$ cycle de l'enseignement secondaire (40\%). Les garçons sont un peu plus nombreux que les filles ( $55 \%$ ). Au niveau de la performance scolaire, $83 \%$ des élèves ont au moins un an de retard scolaire, mais à la différence de la catégorie des " désengagés», $73 \%$ se sont présentés aux épreuves du DNB sans succès. Les boursiers représentent $33 \%$ de ce groupe. Du côté de l'environnement familial, le profil global reste similaire aux décrocheurs " désengagés».

\subsection{Les décrocheurs « attendus »}

Cette dernière catégorie, rassemblant $15 \%$ des décrocheurs, présente des caractéristiques scolaires et socioéconomiques les plus défavorables par rapport aux autres catégories de décrocheurs. Leur décrochage est donc le plus "prévisible ". L'écart entre les filles et les garçons est maximal, comparativement aux trois autres catégories ( $40 \%$ des filles ici seulement). C'est le profil d'élèves scolarisés dans l'enseignement adapté (49\%) et dans les CAP en 2 ans (43\%), qui représentent généralement l'orientation 
réservée aux élèves issus de l'enseignement spécial (3 $3^{\text {ème }}$ SEGPA - Section d'enseignement général et professionnel adapté - ou $3^{\text {ème }}$ Insertion). Ils ont à $95 \%$ au moins un an de retard scolaire, et sont à $99 \%$ non-inscrits aux épreuves du DNB. Cette catégorie regroupe le plus de jeunes issus de familles monoparentales (40\%). L'essentiel de ces jeunes $(80 \%)$ sont issus de milieux défavorisés. Le premier responsable légal de l'élève occupe un emploi dans seulement $43 \%$ des situations et le second responsable légal dans $21 \%$ des cas. Les familles qui ont quatre enfants et plus sont surreprésentées dans cette catégorie de décrocheurs. Ce sont par conséquence des jeunes qui cumulent les handicaps scolaires et sociaux.

\subsection{D'autres profils-types de décrocheurs}

D'autres travaux ont également démontré l'existence de plusieurs types de décrocheurs (Janosz et $a l .$, 2000. Fortin et al., 2006). Plus récemment, en France, Dardier, Laïb et Robert-Bobée (2013), à partir des données du panel DEPP 1995, proposent une typologie des parcours scolaires des élèves décrocheurs. Ils distinguent trois groupes principaux de décrocheurs : le groupe "faible niveau scolaire " (46\% des décrocheurs), composé de jeunes ayant quitté la classe de $3^{\text {ème }}$, une formation menant à un niveau CAP/BEP ou baccalauréat. Le groupe «bons élèves " (33\% des décrocheurs) qui ont un bon niveau scolaire mais ont décroché de leurs études au lycée dans les filières G\&T ou du niveau post CAP/BEP de l'enseignement professionnel. Enfin, le groupe "passage par des classes adaptées au collège " (21\% des décrocheurs) qui ont bénéficié de l'enseignement spécialisé et qui décrochent en fin du $1^{\text {er }}$ cycle de l'enseignement secondaire ou en CAP/BEP.

Cette typologie rejoint en partie les catégories établies sur la base de nos données. En effet, la performance scolaire distingue nettement les décrocheurs "inattendus » des autres catégories, car ils quittent l'école comme les "bon élèves ". Le même rapprochement peut être opéré entre les décrocheurs " attendus " et ceux qui ont fait un "passage par des classes adaptées au collège ".

Notre apport consiste à prendre en compte, à partir de données exhaustives, non seulement les caractéristiques scolaires, mais aussi des éléments qui illustrent le contexte social de l'élève dans l'élaboration d'une typologie des décrocheurs.

Nous montrons que les décrocheurs sont loin de former un ensemble homogène. Si la ligne de démarcation entre la catégorie des "inattendus " et celle des " attendus " est clairement établie, l'existence des deux catégories intermédiaires laisse supposer que, pour ces deux profils de jeunes, le décrochage scolaire est seulement une question de temps, c'est-à-dire qu'il n'a pas lieu au même moment du parcours : soit au moment de la transition entre le premier et le second cycle de l'enseignement secondaire, soit l'année d'achèvement de ce dernier. Au vu de leur faible niveau scolaire, leur passage 
dans l'enseignement professionnel serait une " chance fragile " pour certains, une "élimination différée " pour d'autres ${ }^{7}$ (Felouzis, 2003).

\section{Dissocier l'effet établissement 4 du rôle des caractéristiques individuelles}

Les travaux qui portent sur les effets de contexte et le décrochage scolaire en utilisant des données hiérarchisées et des modélisations multiniveaux sont rares. Aucun n'existe à notre connaissance sur des données françaises ${ }^{8}$.

L'objectif ici est de dissocier les effets des caractéristiques individuelles et familiales des effets propres aux établissements scolaires (Raudenbush et Willms, 1995 ; Lee, 2000).

En traitant l'effet de l'organisation scolaire sur l'absentéisme, Bryk et Thum (1989) concluent notamment à l'existence d'un effet établissement sur le décrochage : la taille de l'établissement, le regard que portent les élèves sur les enseignants, la concentration en classe, la fréquence des problèmes de discipline, la diversité des parcours scolaires et des origines sociales sont autant de variables qui agissent significativement sur l'absentéisme et le décrochage scolaire. Les travaux de Rumberger (1995) et Rumberger et Thomas (2000) mesurent l'impact des attributs des établissements - la composition sociale, les ressources de l'école mesurées notamment par le taux d'encadrement, la taille de l'établissement, son statut public ou privé ou encore sa localisation... - sur le décrochage scolaire, en contrôlant des facteurs individuels.

L'analyse de l'effet du contexte scolaire attribue la différence entre les écoles en termes de décrochage aux caractéristiques sociodémographiques individuelles des élèves et à la composition socio-économique moyenne des établissements scolaires.

Enfin, les travaux de Lee et Burkam (2000) révèlent que l'explication du décrochage scolaire par des caractéristiques individuelles de l'élève est insuffisante. L'établissement scolaire influence le décrochage scolaire, ses effets sont médiatisés par des variables de composition, comme les caractéristiques démographiques du public scolarisé, ou d'organisation, comme la taille de l'établissement, le cycle d'enseignement ou la nature des programmes étudiés.

7. Leur décrochage interviendra un peu plus tard, mais de manière prévisible compte tenu des handicaps scolaires et sociaux qu'ils manifestent et qui les distinguent des autres élèves.

8. On compte en revanche nombre de travaux sur des données françaises dans le champ des sciences de l'éducation qui mobilisent des approches multiniveaux et relient les performances scolaires des élèves aux différents environnements d'apprentissage, qu'ils concernent la classe ou l'établissement scolaire (voir Dumay et Dupriez, 2009 ; Bressoux, 2010). 


\subsection{Facteurs de décrochage scolaire : ce qui relève des établissements scolaires}

L'effet dit " d'établissement » dans l'enseignement professionnel sur le décrochage scolaire semble moins important que celui de l'enseignement G\&T.

Pour l'ensemble des élèves ${ }^{9}$, la part de la variance du risque de décrochage relève, pour $16 \%$, de l'effet dit "d'établissement ». Pour les élèves de CAP, $2.6 \%$ de la variance tient à l'hétérogénéité des seuls établissements scolaires. Pour les élèves préparant un Bac Pro en lycée professionnel (LP), $3.5 \%$ de la variance est liée à l'hétérogénéité des seuls établissements scolaires. Enfin, pour les élèves préparant un baccalauréat G\&T, $9.5 \%$ de la variance relève de l'hétérogénéité des établissements. La relative faiblesse de l'effet « d'établissement » est due au poids prédominant des variables individuelles, et plus largement celles qui concernent directement l'élève lui-même (notion de caractéristiques proximales de Potvin et Pinard, 2012, pour marquer la faible distance entre l'individu et la caractéristique qui le concerne, par opposition aux variables distales ou environnementales qui sont des faibles prédicteurs du décrochage).

En matière de décrochage scolaire, il existe donc une plus grande homogénéité entre les différents lycées professionnels qu'entre les différents lycées G\&T. Cette moins grande variabilité des premiers relève des caractéristiques des publics qu'ils accueillent (plus homogènes que les élèves scolarisés dans le cursus G\&T). Pour autant, le décrochage est plus important au LP qu'au LG\&T.

Comme l'effet " d'établissement " est faible dans les LP, on ne peut en déduire que ces types d'établissements "produisent" du décrochage, mais que les caractéristiques du public qu'ils accueillent font que le décrochage constaté y est plus important. Autrement dit, ce n'est pas l'enseignement professionnel qui a un effet en lui-même sur le décrochage mais ce sont les caractéristiques de son public qui "prédisposeraient " ce type d'enseignement à rencontrer autant de situations de décrochage scolaire (les élèves en difficulté au collège sont ceux orientés le plus souvent en LP). Les critiques adressées alors au LP semblent partiellement injustifiées car l'établissement en tant que tel n'interviendrait pas ou peu sur le décrochage (comparativement au LG\&T). Ce constat est une autre façon de souligner les conséquences de l'orientation par "l'échec " ou l'orientation «subie " par les élèves de la voie professionnelle.

9. Dans cette partie, on exclut les décrocheurs collégiens. Ce choix repose sur deux motifs : le premier est que la plupart des élèves en classe de troisième ont quinze ans et donc ne peuvent pas être décrocheurs du collège, selon la définition institutionnelle (avoir 16 ans au moins). Le second motif est que les élèves décrocheurs du collège (peu nombreux et peu représentatifs du fait du premier motif) ont plus de chances que tous les autres élèves décrocheurs de se voir proposer un autre dispositif de formation et donc finalement de " raccrocher " l'année suivante. 
Par ailleurs, le décrochage scolaire est, toutes choses égales par ailleurs, plus important dans l'enseignement public que privé. Le fait d'être scolarisé dans le secteur public augmente le risque de décrocher partout, sauf pour le cursus CAP, pour lequel enseignement public et privé ne se distinguent pas. La problématique des élèves orientés vers le CAP reste la même quel que soit le secteur d'enseignement et renvoie davantage aux caractéristiques des élèves qu'à une organisation spécifique d'un secteur. Pour l'enseignement G\&T, des politiques d'établissement du secteur privé peuvent conduire à sélectionner des élèves sur la base de leurs résultats scolaires à l'entrée et à se séparer de ceux les plus "à risque de décrocher", pour les renvoyer vers le secteur public, et ainsi préférer des élèves dont la réussite est plus assurée, afin de garder une position attractive.

La taille de l'établissement fréquenté ne joue globalement pas sur le risque de décrochage $^{10}$, alors même qu'Afsa (2014) a établi un lien entre la taille de l'établissement et les performances scolaires des élèves : la taille pourrait donc ici voir son effet en partie capté par les variables des performances individuelles (la taille jouant sur les résultats scolaires qui eux-mêmes expliquent le risque de décrochage).

En termes de composition sociale des établissements (part d'élèves issus de familles de " cadres supérieurs, enseignants ou cadres moyens"), cette caractéristique est sans effet significatif. On peut penser que c'est plutôt le fait d'être dans un établissement où les performances des élèves sont élevées qui l'emporte sur les origines sociales qui le composent (l'origine sociale déterminant pour partie le niveau de performance moyen). En effet, on montre que la note moyenne des élèves de l'établissement au DNB favorise le maintien des élèves de l'établissement et leur poursuite d'études. Pour les élèves pris dans leur ensemble (hors collèges) et pour ceux de l'enseignement G\&T séparément, plus les résultats scolaires en moyenne dans l'établissement sont élevés et plus la probabilité individuelle de décrocher diminue. L'absence de cet effet dans le cursus professionnel tient à la plus grande homogénéité entre établissements en termes de performances des élèves qui les fréquentent.

Concernant enfin les enseignants, leur âge moyen montre que leur expérience professionnelle agit comme facteur de prévention du décrochage pour les élèves pris dans leur ensemble, mais cet effet n'est manifeste que pour les jeunes préparant un CAP. On peut penser qu'avec l'expérience, les enseignants sont mieux à même de prendre en charge les difficultés scolaires, accentuées à ce niveau de formation. Cet effet serait plus marquant pour le public qui en aurait le plus besoin (CAP). Enfin, notons que la part des enseignants à temps partiel dans un établissement est sans lien avec le décrochage.

10. Y compris lorsque cette variable est introduite dans le modèle à l'aide de plusieurs modalités. 


\subsection{Ce qui a trait aux élèves}

Pour l'ensemble des élèves ${ }^{11}$, le risque moyen de décrocher s'élève à $9 \%$. Certaines classes fréquentées apparaissent être plus "à risque " que d'autres, toutes choses égales par ailleurs ${ }^{12}$ : relativement à la seconde générale, le risque est plus faible en première et en terminale G\&T, alors que ce risque est le même pour la seconde professionnelle (toujours par rapport à la seconde générale) mais il est plus faible pour la première professionnelle et plus fort pour la terminale professionnelle. Dans la filière professionnelle, le décrochage s'opère davantage en terminale que lors des deux années antérieures; pour la filière générale, le décrochage, quand il a lieu, est plus probable en seconde qu'au cours des deux années qui suivent.

En ne considérant que les LP et les jeunes préparant un CAP, par rapport à un élève en $1^{\text {ère }}$ année, un jeune de l'année terminale a plus de risque de quitter sa formation sans obtenir un diplôme. Même constat pour le cursus Bac pro, puisqu'un élève de la $2^{\text {nde }}$ et de la $1^{\text {ère }}$ Bac pro a plus de chance de persévérer qu'un élève de l'année terminale. Comment expliquer les différences entre les niveaux de décrochage (classe de terminale ou de seconde) selon la filière professionnelles ou G\&T ?

Dans la filière professionnelle, l'hypothèse est que les décrocheurs de l'année terminale (Bac pro ou CAP) ne sont pas totalement des décrocheurs au sens conventionnel du terme (en retard scolaire, faible résultat scolaire,...) mais surtout des décrocheurs au sens institutionnel : ils quittent le système scolaire certes sans diplôme mais pas sans qualification. On sait qu'en termes d'insertion des sortants du secondaire, il existe un différentiel de gains d'accès à l'emploi et de stabilité de ce dernier pour les élèves qui poursuivent leurs études jusqu’à la dernière année du cursus de l'enseignement secondaire (Arrighi et al., 2009). Cet avantage est à mettre en parallèle avec, d'une part, la probabilité d'obtenir un baccalauréat professionnel trois après l'entrée en $2^{\text {nde }}$ professionnelle (Defresne et Dubois, 2013) et, d'autre part, le sentiment de frustration causé par un redoublement ou une situation d'échec. Cela laisse penser que la non-obtention d'un diplôme ne constitue pas non plus, pour les élèves de niveau terminal, un obstacle à la décision d'arrêter les études et d'entrer dans la vie active (Bernard et Michaut, 2014) ; ils mobilisent à cet effet leurs propres connaissances du monde de l'entreprise pour y avoir déjà travaillé ou effectué un stage dans le cadre de la formation initiale (Barret et al., 2014). Dans la filière G\&T, l'année de seconde reste une année clé pour la poursuite d'études ; c'est en effet une année d'orientation et les décrocheurs y sont les jeunes décrocheurs au sens conventionnel, donc les plus faibles scolairement ( $c f$. infra).

Les élèves en retard scolaire d'une année voient leur probabilité de décrocher augmenter par rapport à ceux qui sont «à l'heure »; les élèves qui ont cumulé au moins deux ans de retard

11. Les collégiens étant toujours exclus.

12. Le tableau des estimations figure en annexe électronique. 
durant leur parcours scolaire voient ce risque s'accroître encore plus fortement. Pour les élèves en LP et qui préparent un CAP, le retard scolaire les pénalise également en termes de décrochage mais moins fortement que si l'on considère l'ensemble des élèves. Quant à ceux qui préparent un baccalauréat professionnel, le constat est identique (cependant, leur retard scolaire représente un désavantage plus grand que pour la population des jeunes en CAP). Pour les élèves scolarisés dans le second cycle de l'enseignement G\&T, ceux " en retard " se distinguent fortement des autres qui sont "à l'heure " quant au risque du décrochage, le retard y étant un caractère plus distinctif encore qu'en LP et aussi pour l'ensemble des élèves.

Globalement, la faiblesse du niveau scolaire est fortement liée au risque de quitter le système éducatif prématurément (Afsa, 2013). On sait par ailleurs que le faible niveau scolaire est fortement dépendant de l'origine sociale de l'élève.

De la même manière, le résultat au DNB est un «bon» indicateur du décrochage. En effet, et logiquement, les élèves qui obtiennent une mention et, dans une moindre mesure, ceux qui réussissent sans mention voient leur probabilité de décrochage diminuer relativement aux élèves qui sont refusés ou encore ceux qui ne se sont pas présentés à l'épreuve. Pour l'académie d'Aix-Marseille (2012-2013), 78 \% des élèves (décrocheurs ou pas) qui ont réussi les épreuves du DNB avec la moyenne ou plus à l'examen sont issus des familles "favorisées », alors qu'ils ne sont que 29 \% issus d'un milieu social "défavorisé ". Reste que les résultats au DNB constituent un puissant déterminant du risque de décrochage, et ce au-delà du fait de leur nature endogène. Les résultats scolaires sont en effet eux-mêmes expliqués par l'origine sociale des élèves, cette dernière expliquant également le décrochage.

Globalement, les garçons sont, tout autant que les filles, toutes choses égales par ailleurs, susceptibles de décrocher lorsque l'on considère l'ensemble des élèves. En retenant uniquement les jeunes qui préparent un baccalauréat (Pro ou G\&T), filles et garçons sont à égalité face de décrochage. En revanche, les garçons ont plus de chances de se maintenir en formation lorsqu'ils sont scolarisés dans un cursus CAP. Les filles y sont sous-représentées, les spécialités CAP tertiaires (service, 22 spécialités pour la $1^{\text {ère }}$ année), plus souvent choisies par elles, sont en effet moins nombreuses que les spécialités industrielles (production, 45 spécialités pour la 1 ère année) " traditionnellement " masculines. L'éventail des choix étant a priori plus restreint pour les spécialités de services, plus demandées par les filles, la demande pour certaines spécialités est alors plus importante que l'offre et les orientations des filles relèveraient plus d'un choix par défaut que pour les garçons. Ces orientations contrariées pouvant alors expliquer la plus forte propension des filles à décrocher en CAP par rapport aux garçons. 


\subsection{Ce qui relève de l'environnement familial}

La structure familiale joue un rôle central. Par rapport à la taille de la fratrie, les enfants de famille (très) nombreuse (avec quatre frères/sœurs et plus) ou avec un enfant unique se voient associer un risque plus élevé de décrochage que les autres. Cet effet est plus net encore pour les élèves d'un cursus G\&T. Pour les jeunes en CAP, la taille de la fratrie est sans effet. Enfin, la monoparentalité de la famille apparait comme un facteur "annonciateur " du décrochage scolaire ; cet effet étant de la même ampleur quel que soit le cursus suivi. Contrairement à la monoparentalité, le fait d'être boursier correspond à un décrochage scolaire en diminution, pour les élèves scolarisés dans le cycle d'enseignement G\&T, cette caractéristique est plus distinctive encore quailleurs, une bourse aidant très probablement les plus défavorisés à se rapprocher, du point de vue de leurs conditions matérielles d'études, des élèves mieux dotés socialement en lycée G\&T.

Concernant la situation vis-à-vis du marché du travail du responsable légal de l'élève, le fait que ce dernier soit en emploi ou ait été actif occupé par le passé et désormais retraité joue un rôle favorable dans la poursuite des études. A contrario, le chômage du père (ou de la mère dans le cas d'une famille monoparentale, le plus souvent) augmente globalement le risque de décrochage. Pour les élèves du LG\&T, ce n'est pas le critère de la situation professionnelle du responsable légal qui distingue les décrocheurs des autres élèves. En revanche, lorsque le responsable légal est au chômage, le risque de décrochage des jeunes en CAP s'accroît davantage que celui des jeunes préparant un Bac pro.

On sait qu' une origine sociale modeste contribue globalement à expliquer la sortie sans diplôme du système éducatif : les enfants d'ouvriers comptent parmi eux quatre fois plus de décrocheurs que les enfants de cadres (Afsa, 2013). Pour les jeunes préparant un CAP dans l'académie d'Aix-Marseille, l'origine sociale n'explique pas, à elle seule, leur décrochage ; c'est la situation des parents vis-à-vis du marché du travail qui est déterminante. Ces jeunes partagent en effet une origine sociale globalement " défavorisée "; c'est le lien avec l'activité professionnelle des parents (ou du parent) qui fait la différence entre les décrocheurs et les autres élèves. Le fait d'avoir un parent en emploi protège alors du décrochage scolaire.

En revanche, dans le cursus $G \& T$, ni la catégorie socioprofessionnelle ni la situation sur le marché du travail ne sont déterminantes pour expliquer le décrochage. Pour cette population, les facteurs clés sont liés aux performances scolaires et à la structure familiale. Enfin, pour les jeunes dans une filière de Bac pro, être d'origine sociale " favorisée " protège du risque de décrochage et vient se cumuler au rôle joué par la situation d'emploi du parent.

Rappelons que ces résultats distinguent ce qui relève des élèves et de leurs familles de ce qui tient aux caractéristiques des établissements fréquentés. Cette approche permet de montrer que les performances scolaires (y compris les performances moyennes mesu- 
rées au niveau des établissements fréquentés) constituent, pour tous les élèves, de forts indicateurs du décrochage scolaire. Ce constat a déjà été mis en évidence dans des travaux précédents mais pour la France ; les analyses menées jusqu’ici n’isolaient pas les effets établissements du rôle des caractéristiques des élèves ${ }^{13}$.

\section{Conclusion}

En dressant le portrait des décrocheurs de l'académie d'Aix-Marseille, on montre ici que le décrochage scolaire peut concerner tout type d'èlève et qu'il atteint globalement $9.3 \%$. On met également en évidence l'existence de plusieurs catégories de décrocheurs, en prenant en compte non seulement la dimension scolaire, mais aussi la dimension sociale des caractéristiques des élèves.

D’un côté, des décrocheurs qui ressemblent fortement aux non-décrocheurs, scolairement performants et socialement "favorisés ", et dont la "carrière " scolaire avait a priori vocation à être longue. D'un autre côté, des élèves qui accumulent les handicaps scolaires et sociaux, plus que les autres. Notre apport relève sur ce point de la qualité des données mobilisées, qui sont des données administratives exhaustives et contenant de nombreuses informations pertinentes.

L'originalité de cet article tient également à la mise en lumière de facteurs relatifs au contexte scolaire et qui agissent indépendamment des caractéristiques des élèves sur la propension de ces derniers à décrocher. Nous mettons en évidence l'existence de moments de la scolarité plus propices au décrochage scolaire que d'autres. S'agissant de l'élève, son retard scolaire et son faible niveau de performance constituent des facteurs prédictifs du décrochage qui opèrent différemment selon le cursus suivi. Quant à la situation familiale, être boursier et enfant d'une famille monoparentale sont des facteurs qui jouent également en faveur du décrochage scolaire.

Par ailleurs, nous mettons en évidence que, une fois contrôlées les caractéristiques des élèves, l'effet de l'établissement scolaire fréquenté est plus important dans les lycées généraux et technologiques qu'au sein des lycées professionnels, alors que le décrochage scolaire est sensiblement plus faible dans les premiers qu'au sein des seconds.

Réduire le plus fort taux de décrochage dans le cursus professionnel passe alors certainement par l'élévation du niveau scolaire des élèves qui s'y trouvent. Le public de cette filière d'enseignement reste en effet faiblement doté en capital scolaire et social, caractéristiques déterminantes pour une poursuite d'études et explicatives du fort taux de décrochage au lycée professionnel.

13. Pour l'ensemble des élèves comme pour les élèves de chacun des cursus distingués, la modélisation multiniveaux est préférable, au sens statistique, à un modèle Logit classique. 
Dans le même ordre d'idées, la composition sociale de l'établissement scolaire n'a aucun effet sur le décrochage scolaire des élèves ; ce résultat est à mettre en lien avec l'homogénéité en matière d'origines sociales des jeunes dans les LG\&T, d'un côté, et dans les LP, de l'autre. A contrario, les établissements se distinguent en matière de performance scolaire des élèves qu'ils accueillent : un élève fréquentant un établissement où la note moyenne au DNB est élevée aura un risque moindre de décrocher, toutes choses égales par ailleurs, que dans un autre établissement où le niveau est plus faible. Dans la mesure où le niveau social défavorisé des élèves joue plutôt en faveur de leur orientation vers le cursus CAP ou Bac pro, que le décrochage est lui-même largement déterminé par ces caractéristiques, l'établissement scolaire (notamment les lycées professionnels) semble alors impuissant à neutraliser l'influence de l'origine sociale sur la performance scolaire et la poursuite d'études (Moreau, 1996).

$\mathrm{Au}$ moment où les 30 ans du Bac pro sont célébrés, et six ans après la réforme censée « démocratiser » l'accès à la certification (rénovation de la voie professionnelle), les premiers bilans révèlent que le passage au Bac pro en 3 ans augmente significativement les chances d'obtention du baccalauréat, ces chances variant sensiblement selon les spécialités de formation (Defresne et Dubois, 2013). Au-delà de l'obtention d'un diplôme de niveau IV, la poursuite d'études dans l'enseignement supérieur d'un bachelier professionnel dépend aussi du positionnement de la spécialité choisie par rapport aux autres spécialités et de la possibilité d'insertion professionnelle associée (Bernard et Troger, 2015). On pourrait s'interroger alors sur l'effet de la filière de formation, notamment dans l'enseignement professionnel, sur le risque individuel de décrocher. Une question à corréler avec les effets structurants de l'offre de formation et son mode de régulation par les acteurs (Berthet et al., 2010), et plus largement avec la répartition de la fonction économique d'un territoire.

Enfin, au-delà de l'effet dit « d'établissement » et d'un effet propre à la spécialité de formation ou du territoire, rappelons que les conclusions de cet article sont propres à l'académie d'Aix-Marseille et donc contingentes à son territoire géographique.

En outre, les données quantitatives dont nous disposons contraignent à considérer le décrochage scolaire comme un phénomène constaté à un moment donné. Or, la littérature rappelle le caractère processuel du décrochage en insistant sur l'interaction, d'intensité différente, entre des facteurs individuels, des facteurs scolaires et des facteurs sociaux.

Chaque situation de décrochage scolaire est ainsi l'aboutissement d'un processus singulier, mais l'issue de ce processus renvoie à des déterminants propres que cet article a mis en évidence.

\section{Bibliographie}

Afsa C. (2013), Qui décroche ? Éducation et formations, (84), pp. 9-20.

Afsa C. (2014), Une question de taille. Éducation et formations, (85), p. 63. 
Arrighi J.-J., Gasquet C. \& Joseph O. (2009), «L'insertion des sortants de l'enseignement secondaire ", Céreq, Note Emploi Formation, (42).

Archambault I. \& Janosz M. (2009), "Fidélité, validité discriminante et prédictive de l'indice de prédiction du décrochage ", Canadian Journal of Behavioural Science/Revue canadienne des sciences du comportement, 41(3), 187.

Barret C., Ryk F. \& Volle N. (2014), « Face à la crise, le fossé se creuse entre niveaux de diplôme ", Bref-Céreq, 319.

Bénabou R. \& Tirole J. (2004), "Willpower and personal rules", Journal of Political Economy, 112(4), pp. 848-886.

Bernard P.-Y. (2011), Le décrochage scolaire (1ère édition), Paris, PUF.

Bernard P.-Y. \& Michaut C. (2014), «Marre de l'école » : les motifs de décrochage scolaire, Notes du CREN, (17).

Bernard P.-Y. \& Troger V. (2015), « Les lycéens professionnels et la réforme du bac pro en trois ans: nouveau contexte, nouveaux parcours? ", Formation Emploi, 131, pp. 23-40.

Berthet T., Dechezelles S., Gouin R. \& Simon V. (2010), « La place des dynamiques territoriales dans la régulation de l'orientation scolaire ", Formation Emploi, 109, pp. 37-52.

Bonnéry S. (2007). Comprendre l'échec scolaire : Élèves en difficultés et dispositifs pédagogiques, Paris, La Dispute.

Boudesseul G., Caro P., Grelet Y. \& Vivent C. (2014), Atlas académique des risques sociaux d'échec scolaire : l'exemple du décrochage, Céreq/DEPP.

Bressoux P. (2010, 2e éd.), Modélisation statistique appliquée aux sciences sociales (1ère éd., 2008), Bruxelles, De Boeck.

Broccolichi S. \& Larguèze B. (1996), «Les sorties sans qualification moins de cinq ans après l'entrée au collège ", Éducation et formations, (48), pp. 81-102.

Bryk A. S. \& Thum Y. M. (1989), "The effects of high school organization on dropping out: An exploratory investigation", American Educational research journal, 26(3), pp. 353-383.

Caille J.-P. (2000), « Qui sort sans qualification du système éducatif ? ", Éducation et formations, (57), pp. 19-37.

Conseil de l'Union Européenne. (2009), "Conclusions du Conseil du 12 mai 2009 concernant un cadre stratégique pour la coopération européenne dans le domaine de l'éducation et de la formation ("Éducation et formation 2020") ", Journal officiel de l'Union européenne, (28).

Dardier A., Laïb N. \& Robert-Bobée I. (2013), « Les décrocheurs du système éducatif : de qui parle-t-on. France », Portrait social, INSEE. 
Defresne F. \& Dubois M. (2013), « Le baccalauréat professionnel en trois ans après la troisième : vers une élévation du niveau des élèves ? ", Éducation et formations, (84), pp. 61-69.

Dumay X. \& Dupriez V. (dir.), (2009), L'efficacité dans l'enseignement. Promesses et zones d'ombre, Bruxelles, De Boeck, 292 p.

Dumay X. \& Dupriez V. (2004), «Effet établissement : effet de processus et/ou effet de composition?", Les cahiers de recherche en éducation et formation, (36), pp. 3-20.

Esterle-Hedibel M. (2003), Les arrêts de scolarité avant 16 ans : étude des processus (Roubaix, Nord), Rapport de recherche, CESDIP/CNRS, IUFM Nord-Pas-de-Calais, FASILD Nord-Pas-de-Calais.

Felouzis G. (2003), "La ségrégation ethnique au collège et ses conséquences ", Revue française de sociologie, 44(3), pp. 413-447.

Fortin L., Royer É., Potvin P., Marcotte D. \& Yergeau É. (2004), « La prédiction du risque de décrochage scolaire au secondaire : facteurs personnels, familiaux et scolaires", Canadian Journal of Behavioural Science/Revue canadienne des sciences du comportement, 36(3), 219.

Fortin L., Marcotte D., Potvin P., Royer É. \& Joly J. (2006), “Typology of students at risk of dropping out of school: Description by personal, family and school factors", European Journal of Psychology of education, 21(4), pp. 363-383.

Glasmann D. \& Oeuvrard F. (2004), La déscolarisation, Paris, La Dispute.

Janosz M., Le Blanc M., Boulerice B., \& Tremblay R. E. (2000), "Predicting different types of school dropouts: A typological approach with two longitudinal samples", Journal of educational psychology, 92(1), 171.

Lee V. E. (2000), "Using hierarchical linear modeling to study social contexts: The case of school effects", Educational Psychologist, 35(2), pp. 125-141.

Lee V. E. \& Burkam D. T. (2000), “Dropping out of high school: The role of school organization and structure", American Educational Research Journal, 40(2), pp. 353-393.

Marc F. (dir) (2013), Moyens des politiques publiques et dispositions spéciales. Rapport général fait au nom de la Commission des Finances sur le projet de loi de finances pour 2014, adopté par l'Assemblée nationale, Tome 3, annexe 14.

McNeal Jr R. B. (1997), "High school dropouts: A closer examination of school effects", Social Science Quarterly, pp. 209-222.

Millet M. \& Thin D. (2005), Ruptures scolaires: l'école à l'épreuve de la question sociale, Collection «Le lien social », PUF, Paris. 
Ministère de l'Éducation nationale, de l'Enseignement supérieur et de la Recherche (DEPP), (2014), Géographie de l'école (11).

Moreau L. (1996), La pauvreté et le décrochage scolaire ou la spirale de l'exclusion. Direction de la recherche, de l'évaluation et de la statistique. [ministère de la Sécurité du revenu].

Parker J. G. \& Asher S. R. (1987), " Peer relations and later personal adjustment: Are low-accepted children at risk?", Psychological bulletin, 102(3), 357.

Potvin P. \& Pinard R. (2012), « Deux grandes approches au Québec en prévention du décrochage scolaire : l'approche scolaire et l'approche communautaire ", Gilles J.-L, Potvin P. \& Tièche-Christinat C. (dir.), Les alliances éducatives pour lutter contre le décrochage scolaire, Bern, Peter Lang, pp. 129-147.

Raudenbush S. W. \& Willms J. (1995), "The estimation of school effects", Journal of educational and behavioral statistics, 20(4), pp. 307-335.

Rumberger, R. W. (1987), "High school dropouts: A review of issues and evidence", Review of educational research, 57(2), pp. 101-121.

Rumberger R. W. (1995), "Dropping out of middle school: A multilevel analysis of students and schools", American educational Research journal, 32(3), pp. 583-625.

Rumberger R. W. (2004), "Why Students Drop Out of School”, in Orfield G. Dropouts in America: Confronting the graduation rate crisis, Harvard Educational Pub Group, pp. 131-155.

Rumberger R. W. \& Thomas S. L. (2000), "The distribution of dropout and turnover rates among urban and suburban high schools", Sociology of Education, pp. 39-67.

Sweeten G., Bushway S. D. \& Paternoster R. (2009), "Does dropping out of school mean dropping into delinquency?”, Criminology, 47(1), pp. 47-91.

Vitaro F., Larocque D., Janosz M. \& Tremblay R. E. (2001), "Negative social experiences and dropping out of school", Educational Psychology, 21(4), pp. 401-415.

Wang M. C., Haertel G. D \& Walberg H. J. (1990), "What influences learning? A content analysis of review literature", The Journal of Educational Research, 84(1), pp. 30-43.

Weixler F. (2014), Évaluation partenariale de la politique de lutte contre le décrochage scolaire, Rapport de diagnostic, 28 mars, ministère de l'Éducation nationale. 